\title{
The effects of variations in the interpellet interval on wheel running in the rat
}

\author{
ANTHONY L. RILEY \\ The American University, Washington, D.C. \\ CORA LEE WETHERINGTON \\ University of North Carolina, Charlotte, North Carolina \\ and \\ ANDREW R. DELAMATER, DAVID B. PEELE, and ROBERT J. DACANAY \\ The American University, Washington, D.C.
}

\begin{abstract}
Three rats were given access to a running wheel during spaced food deliveries. As the interpellet interval increased, the overall amount of running increased, the rate of running neither systematically increased nor decreased, and the locus of maximal running occurred later in the interpellet interval. Both the overall amount asd the temporal distribution of running exhibited considerable within-schedule changes during successive exposures to the different schedules. These data are discussed in terms of Staddon's (1977) assignment of behavior into different functional classes, that is, schedule-induced and facultative.
\end{abstract}

Following the initial demonstration of excessive drinking under schedules of spaced food delivery, that is, schedule-induced polydipsia (SIP; Falk, 1961), a wide range of manipulations have been examined for their effects on its development and maintenance (see Falk, 1966, 1971; Freed, Zec, \& Mendelson, 1978; Roper, 1981; Staddon, 1977; Wetherington, 1982). One manipulation that has received considerable attention is the actual schedule of food delivery itself (Falk, 1966, 1971; Hawkins, Schrot, Githens, \& Everett, 1972; Roper, 1980; Staddon, 1977; Wetherington, 1979). The typical finding is that as the interval between pellet deliveries increases, the amount of water consumed increases, but with further increases in the interpellet interval (IPI), it decreases, that is, polydipsic consumption is an inverted-U-shaped function of the IPI.

Although SIP and other schedule-induced behaviors are clearly affected by changes in the interfood interval (see Flory, 1969; Innis, Simmelhag-Grant, \& Staddon, 1983; Roper, 1980), it is unclear whether behavior that is not induced by spaced food deliveries is similarly affected. During spaced food deliveries, a number of behaviors occur that are not increased in frequency by pellet delivery, that is, they are not schedule induced, but instead simply have a nonzero baseline of occurrence in food-

Requests for reprints should be sent either to Anthony L. Riley, Psychopharmacology Laboratory, Department of Psychology, The American University, Washington, DC 20016, or to Cora Lee Wetherington, Department of Psychology, University of North Carolina at Charlotte, Charlotte, NC 28223. Andrew R. Delamater is at Dalhousie University, Halifax, Nova Scotia, Canada, and David B. Peele is at the Environmental Protection Agency, Rescarch Triangle Park. North Caralina. deprived animals (see Roper, 1981; Staddon, 1977; Staddon \& Ayres, 1975). This group of non-schedule-induced behaviors has been termed "facultative" by Staddon (1977). One such behavior that has been extensively investigated in the rat is wheel running (Levitsky \& Collier, 1968; Penney \& Schull, 1977; Riley, Peele, Richard, \& Kulkosky, 1981; Roper, 1978; Segal, 1969; Staddon, 1977; Staddon \& Ayres, 1975; Wetherington, Brownstein, \& Shull, 1977). Although there are several reports on the occurrence of wheel running under schedules of spaced food delivery, only a single study has systematically examined changes in wheel running as a function of variations in IPI (Staddon, 1977). In that report, rats' wheel running was examined under IPI values of 5 , $10,30,60$, and $180 \mathrm{sec}$. Similar to the typical finding with SIP, the amount of wheel running initially increased as the IPI value increased. Unlike SIP, however, running did not decrease with further increases in the IPI, but instead continued to increase. The effect of IPI on rate of wheel running was also different from its effects on SIP. Although rate of polydipsic drinking decreases with IPIs longer than about $20 \mathrm{sec}$ (Hawkins et al., 1972; Heyman \& Bouzas, 1980; Roper, 1980; Staddon, 1977; Wetherington, 1979), Staddon (1977) found that rate of wheei nunning increased with IPI value.

Although it appears that SIP and wheel running are differentially affected by variations in the IPI, an effect consistent with their differential classification by Staddon (1977) as schedule-induced and facultative, there is at least one factor that may qualify such a conclusion. Specifically, in Staddon's (1977) report, the effects of IPI value on wheel running were assessed in rats given concurrent access to both a running wheel and water. It is unknown 
to what extent, if any, the opportunity to drink affected the display of wheel running or its functional relationship to changes in the IPI. Staddon (1977) suggested that the changes in running that occurred with changes in the IPI were due in part to temporal competition from the concurrent changes in drinking. Evidence for interaction between running and drinking has, in fact, been demonstrated by Penney and Schull (1977), Riley et al. (1981), Roper (1978), and Staddon and Ayres (1975).

The effects of variations in IPI on wheel running in the absence of SIP, therefore, are unknown. To address this question, the present experiment examined wheel running in the rat without concurrent access to water under four IPI values ranging from 30 to $360 \mathrm{sec}$. In addition to examining the amount and rate of running, the postpellet temporal distribution of running was also examined.

\section{METHOD}

\section{Subjects}

The subjects were three experimentally naive female rats of LongEvans descent, approximately 90 days of age at the beginning of the experiment. They were housed in individual wire-mesh cages. The housing quarters were maintained on a 12 -h-light/12-h-dark cycle and at an ambient temperature of $23-24^{\circ} \mathrm{C}$.

\section{Apparatus}

A single apparatus was used for the daily experimental sessions. This apparatus consisted of a running wheel, $60 \mathrm{~cm}$ in diameter and $9 \mathrm{~cm}$ wide, with a small $11.5 \times 5.5 \times 15.5 \mathrm{~cm}$ entrance-way chamber attached to its lower front wall. A food tray was centered on the door of the chamber, $3.5 \mathrm{~cm}$ above the floor. A No. 1829 24-V dc lamp was located directly above the food tray. The back wall of the running wheel was constructed of 1 -mm-thick aluminum; the front wall of the wheel and the attached chamber were constructed of 3-mm-thick Plexiglas; and the floors of the wheel and chamber were made of 6-mm hardware cloth.

\section{Procedure}

Following food deprivation and reduction to $85 \%$ of ad-lib body weight, the subjects were given daily sessions consisting of the delivery of a single $45-\mathrm{mg}$ Noyes food pellet delivered independently of their behavior once every $60 \mathrm{sec}$, that is, a fixed-time (FT) 60 -sec schedule, for a total of 30 pellet deliveries. This schedule was in effect for 21-24 days. Following exposure to the FT 60, all subjects were run under three additional FT values: FT 30, FT 180 ,

Table 1

The Sequence of Exposure of Individual Subjects to the FT Schedules and the Number of Sessions for Each Schedule

\begin{tabular}{ccc}
\hline Subject & FT Schedule & $\begin{array}{c}\text { Number of } \\
\text { Sessions }\end{array}$ \\
\hline 2 & 60 & 24 \\
& 180 & 21 \\
& 30 & 21 \\
& 360 & 24 \\
4 & 60 & 23 \\
& 30 & 21 \\
& 180 & 21 \\
7 & 360 & 21 \\
& 60 & 21 \\
& 360 & 21 \\
& 180 & 21 \\
\hline
\end{tabular}

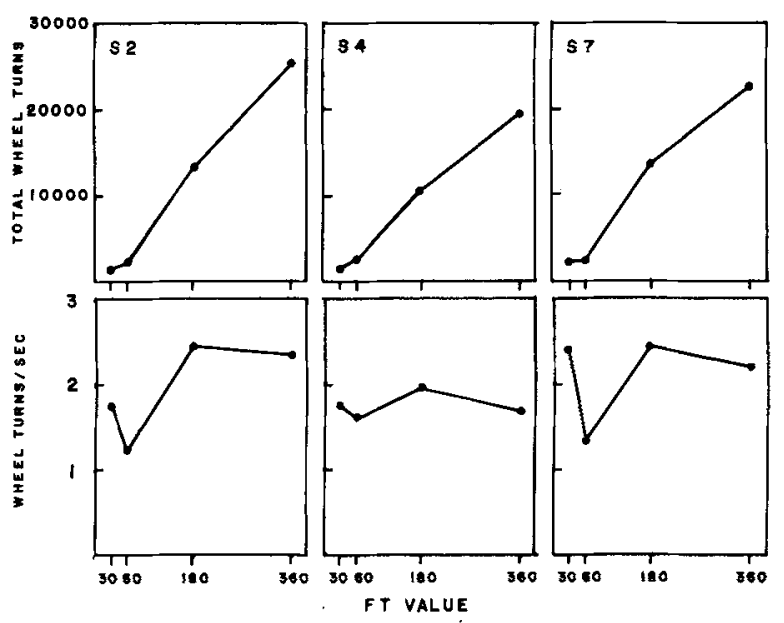

Figure 1. Upper panel: The mean number of wheel turns for each subject under each of the four FT schedules. Lower panel: The mean rate of running per session for each subject under each of the four FT schedules.

and FT 360. As above, under each schedule condition, the session terminated after the delivery of 30 pellets. The specific sequence of the schedules and the number of days the individual subjects were maintained on each schedule are presented in Table 1.

Rotations of the wheel were recorded by a microswitch which was deflected by eight equally spaced notches in the axle of the wheel. A torque of approximately $6.0 \mathrm{~J}$ applied in either direction was sufficient to initiate rotation of the wheel. Immediately following the delivery of each food pellet, wheel runs (1/8 rotations of the wheel) were collected in six consecutive $10-\mathrm{sec}$ intervals. Under the FT 180 and FT 360 schedules, all runs occurring after $50 \mathrm{sec}$ were accumulated in the sixth bin. All programming and recording were accomplished by standard electromechanical devices.

\section{RESULTS}

\section{Overall Amount of Wheel Running}

The upper panel of Figure 1 shows the mean wheel turns per session for each rat for the last 10 days of each of the four FT schedules. For both the FT 30 and FT 60 schedules, there were fewer than 3,000 turns per session, with running under the FT 60 schedule only slightly more than that under the FT 30 schedule. With further increases in the FT value, wheel running increased, yielding approximately 13,000 and 22,000 turns for the FT 180 and FT 360 conditions, respectively. These changes were directly related to the time available under the various schedules. For example, running was 7.1 times greater (range 6.4-8.3) under the FT 180 schedule than under FT 30, and was 12.9 times greater (range 10.6-16.0) under the FT 360 schedule than under FT 30 . If running were perfectly related to the amount of time available under the various schedules, one would expect running under the FT 180 and FT 360 schedules to be 6 and 12 times greater than that under the FT 30 , respectively. This strong relationship between wheel turns and FT value is further corroborated by linear regression coefficients of $.997, .997$, and .988 , respectively, for Subjects 2, 4, and 7.

The lower panel of Figure 1 shows the rate of wheel 


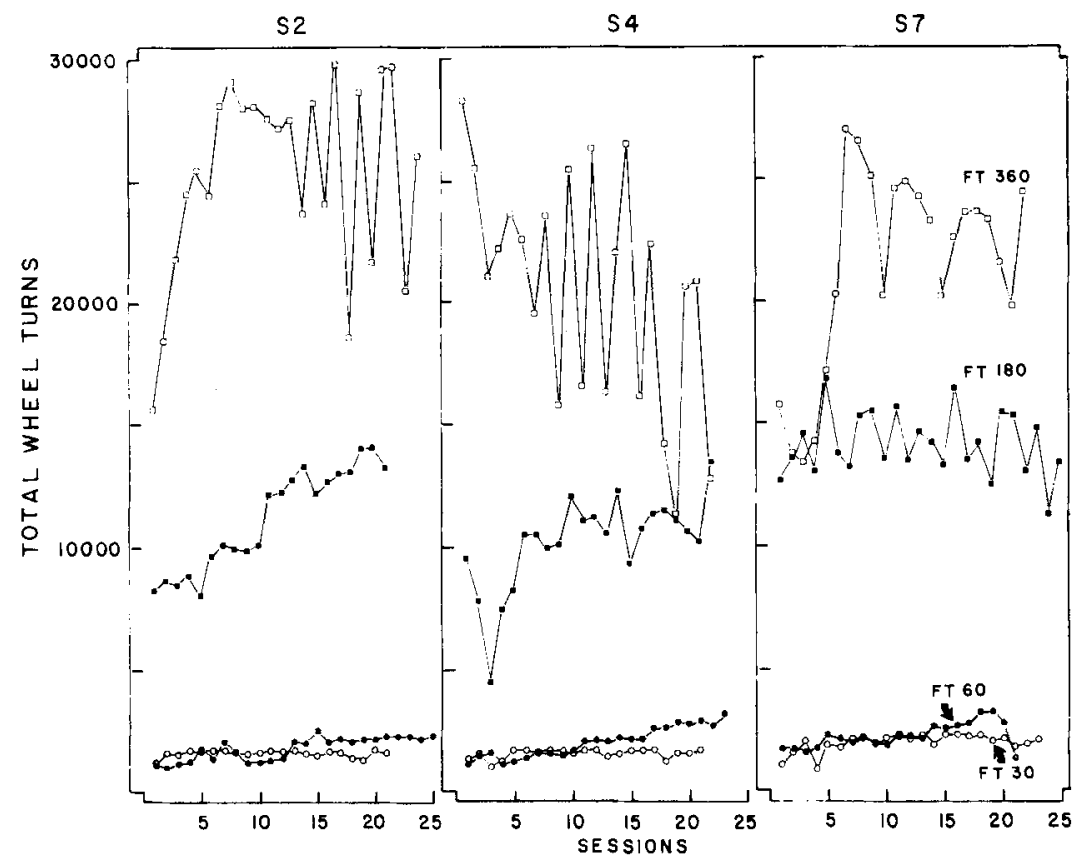

Figure 2. The total number of wheel turns per session for each subject over sessions within each of the four FT schedules.

running as a function of FT value. This measure varied from 1.37 to 2.3 turns (i.e., $1 / 8$ rotations) per second. Although it neither increased nor decreased with FT value, there were several regularities across all rats: (1) The rate was highest under FT 180, (2) the rate was higher under FT 360 than under FT 30 or FT 60 , and (3) the rate was lowest under FT 60 . This last effect may be an order effect, since the FT 60 schedule was the first to which all the rats were exposed. Order effects for the other data points, however, can be ruled out, since, after the FT 60 schedule, order of exposure was randomized.

Figure 2 shows the total wheel turns for each rat for each of the sessions within each FT condition. Examination of the session-by-session changes reveals considerable variability in the development of running to its steady state. For all rats, running under the FT 60 and FT 360 schedules developed gradually. Under the FT 180 schedule, running developed gradually for Subjects 2 and 4 , but very rapidly for Subject 7 . This difference appeared to depend upon whether the prior schedule was longer or shorter: For Subjects 2 and 4, the FT 180 schedule was preceded by a shorter FT value, whereas for Subject 7, the preceding FT was longer. Under the FT 30 schedule, running reached its steady-state level immediately for all rats. These variations in the time course of the within-condition development of running are particularly interesting in view of the linear relationships between wheel turns and FT value shown in Figure 1.

\section{Temporal Distribution of Wheel Running}

Figure 3 shows the postpellet temporal distribution of wheel running for each rat averaged over the last 10 days of each FT schedule. These distributions were affected by the FT value in two major ways. First, running in the first and second 10-sec bins decreased as the FT value increased (the two exceptions were the Bin 2, FT 180 data point for Rat 2 and the Bin 1, FT 60 data point for Rat 7).

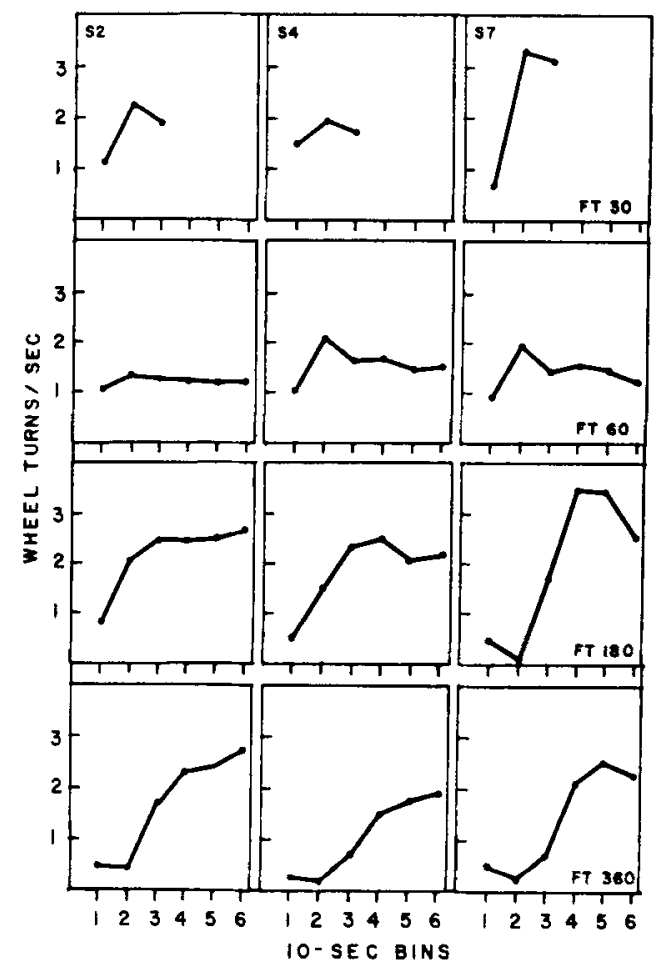

Figure 3. The postpellet temporal distribution of wheel turns for each subject under each of the four FT schedules. Under the FT 180 and FT 360 schedules, the sixth bin reflects postfood time of $50 \mathrm{sec}$ and greater. 
A second effect of the FT schedule was on the locus of maximal running. For all rats, maximal running under the FT 30 and FT 60 schedules was in the second $10-\mathrm{sec}$ bin; as the FT value increased, it occurred later in the interval. For the FT 180 schedule, maximal running occurred in the fourth (Subjects 4 and 7) and sixth (Subject 2) bins. For the FT 360 schedule, it occurred in the fifth (Subject 7) and sixth (Subjects 2 and 4) bins. Because the sixth bin reflects the wheel runs for all postfood time equal to or greater than $50 \mathrm{sec}$, it is difficult to determine the locus of maximal running for those three cases under the FT 180 and FT 360 conditions in which the peak occurred in the sixth bin. The locus of maximal running could have actually occurred during the fifth bin, that is, 40 to $50 \mathrm{sec}$ postfood, during 50 to $60 \mathrm{sec}$ postfood, or later. The general shape of the functions, however, does suggest that the locus was between 40 and $60 \mathrm{sec}$.

Figure 4 shows the bin containing the largest number of wheel turns for each session of each FT condition for each rat. During the FT 30 and FT 360 schedules for Subject 2 , the development of the locus of maximal running to its steady state was virtually immediate. Examination of the remaining 10 curves shows that the development of the terminal locus of maximal running occurred in two or three relatively distinct phases. The changes in the temporal distribution of running were characterized by a decrease in the variability in the locus of maximal running (Subject 4, FT 30, FT 60, and FT 180; Subject 7, FT 360), sometimes involving a shift to a new locus (Subject 2, FT 60 and FT 180; Subject 7, FT 60). In two instances, however, shifts in the locus of peak running occurred without a decrease in variability (Subject 7, FT 30 and FT 180). Careful comparison of these curves with those in Figure 2 reveals no obvious correlation between the changes in total wheel turns and changes in the temporal location of maximal wheel running.

\section{DISCUSSION}

The increase in the amount of wheel running with increases in the FT value is consistent with the aforementioned report by Staddon (1977), in which running and drinking were concurrently available while the IPI was varied from 5 to $180 \mathrm{sec}$. The present research extends the direct relationship between running and IPI value to $360 \mathrm{sec}$, and demonstrates that this relationship is not dependent upon the presence of concurrent drinking.

The differential effect of IPI value on the amount of wheel running and SIP supports Staddon's assignment of running and drinking into differential functional classes of behavior (Staddon, 1977; Staddon \& Ayres, 1975). Although the basis for the inverted-U-shaped relationship between SIP and IPI value is unclear, the fact that wheel running increases with the IPI value is consistent with Staddon's view that wheel running, as a facultative behavior, fills in time or "fits in at times when the tendency to engage in the two dominant classes of activity [i.e., interim and terminal] is weak" (Staddon, 1977, p. 113). From this perspective, wheel running increased as the FT value increased simply because there was more time available in which to run. It should be emphasized, however, that Staddon's (1977) facultative account of wheel running involves the assumption of unmeasured scheduleinduced behavior. In the previous report of a linear relationship between running and IPI (Staddon, 1977), SIP was concurrent with wheel running so that systematic changes in the amount of wheel running as a function of the IPI could be attributed to systematic changes in the amount of SIP. In the present experiment, however, there were no observations, measured or unmeasured, of any scheduled-induced behavior. The consistency of the present data with Staddon's (1977) facultative view, therefore, rests upon the assumption of inferred scheduleinduced behavior. Given Roper's (1981) claim that the

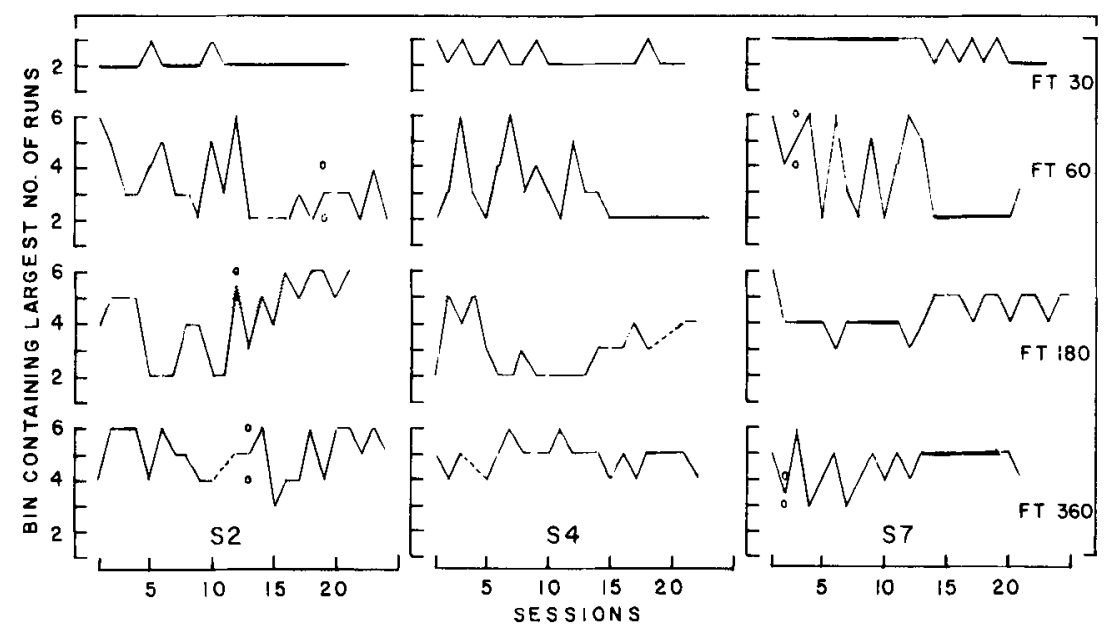

Figure 4. The bin containing the largest number of runs for each subject over sessions within each of the four FT schedules. Circles represent cases in which maximal running occurred in two bins. Dashed lines represent missing data. 
number of behaviors induced by intermittent food is quite limited, this assumption must be subjected to further empirical test.

In Staddon's (1977) report of wheel running, rate of wheel running systematically increased from approximately 0.75 to 25 responses/min as the FT value was varied from 5 to $180 \mathrm{sec}$. In the present experiment, however, rate clearly did not increase with FT value. Staddon (1977) suggested that the relationship he observed was due in part to the accompanying decrease in drinking rate as a function of the FT value. This interpretation implies that, in the absence of drinking, the rate of running might be unaffected by the FT value. The present data on rate of running are somewhat ambiguous on this point and are subject to two descriptions. If the data point from the FT 60 schedule is excluded on the basis of a possible order effect, then there is a considerable reduction in the range of the rate of running ( 1.93 to 2.3 vs. 1.37 to 2.3 turns/ $\mathrm{sec}$ ), suggesting that, indeed, rate may have been un' affected by the FT value. Yet, an important regularity would remain: For all rats, the rate was highest under FT 180, suggesting that rate is a bitonic function of the FT value. This latter description of the data would not necessarily be at odds with the effect Staddon (1977) reported, since FT 180 was the longest value he tested. Perhaps, with longer FT values, rate would have declined. Clearly, more parametric testing of long FTs is required before definitive statements can be made regarding the effect of FT value on running rate, either in the presence of SIP or in its absence.

Taken together, Staddon's (1977) data and the present data indicate the following generalizations regarding the effect of IPI on the amount and rate of wheel running. The total number of wheel turns increases with IPI value regardless of the presence or absence of SIP. The effect of the presence or absence of SIP on rate of running is unclear, but two possibilities emerge: (1) Rate of running increases with IPI value in the presence of SIP, but in its absence is relatively constant, or (2) rate or running is a bitonic function of IPI value regardless of the presence or absence of SIP.

Within each FT condition, wheel running displayed a clear postpellet temporal patterning. In Staddon's (1977) view of the facultative nature of wheel running, this postpellet distribution is determined by other behaviors occurring within the IPI. Immediately following pellet delivery, schedule-induced behaviors presumably compete with wheel running, thus causing it to have a low baseline of occurrence during this period. Near the end of the IPI, as the delivery of food becomes imminent, food-anticipatory behaviors (or terminal behaviors, in Staddon's terminology) successfully compete with wheel running and accordingly reduce the level of wheel running during this period. Wheel running occurs during the intervals between these extremes presumably because no other behaviors, induced or facultative, are present which could or do compete with wheel running. This view adequately accounts for the postpellet temporal distribution of wheel running; however, in the absence of the measurement of these presumed competing schedule-induced behaviors, their occurrence can only be assumed. Furthermore, to account for the change in the distribution of wheel running with increases in the FT value, one must assume a corresponding change in the temporal distribution of these competing behaviors.

Although wheel running clearly is not schedule induced (Penney \& Schull, 1977; Staddon \& Ayres, 1975; Wetherington et al., 1977), it is clear that more attention needs to be directed towards elucidating the conditions under which it occurs and the factors responsible for its distribution in time. Given that the term "facultative behavior" carries considerable theoretical weight, for example, its occurrence is limited and its temporal distribution is defined by concurrently occurring schedule-induced behaviors (see Staddon, 1977), it may be more parsimonious at this time simply to describe behaviors such as wheel running as noninduced (see Wetherington \& Brownstein, 1982). Although SIP and wheel running do appear to belong to different classes of behavior, that is, induced and noninduced, respectively, it should be noted that there are temporal similarities in how each of these behaviors is affected by changes in the IPI. In the present research, the temporal distribution of running was displaced into the IPI as the FT value increased. This effect has also been reported to occur for SIP (see Rosellini \& Burdette, 1980; Shurtleff, Delamater, \& Riley, 1983). This similar effect on the temporal distribution of behavior suggests that the temporal modulating effect of the schedule is more fundamental than schedule induction.

\section{REFERENCES}

FALK, J. L. (1961). Production of polydipsia in normal rats by an intermittent food schedule. Science, 133, 195-196.

FALK, J. L. (1966). Schedule-induced polydipsia as a function of fixed interval length. Journal of the Experimental Analysis of Behavior, 9 , 37-39.

FALK, J. L. (1971). The nature and determinants of adjunctive behavior. Physiology \& Behavior, 6, 577-588.

FloRY, R. (1969). Attack behavior as a function of minimum inter-food interval. Journal of the Experimental Analysis of Behavior, 12, 825-828.

Freed, W. J., Zec, R. F., \& MENDELson, J. (1978). Schedule-induced polydipsia: The role of orolingual factors and a new hypothesis. In J.A.W.M. Weijnen \& J. Mendelson (Eds.), Drinking behavior: Oral stimulation, reinforcement and preference. New York: Plenum Press.

Hawkins, T. D., Schrot, J. F., Githens, S. H., \& Everett, P. B. (1972). Schedule-induced water and alcohol ingestion. In R. M. Gilbert \& J. D. Keehn (Eds.), Schedule effects: Drugs, drinking and aggression. Toronto: University of Toronto Press.

Heyman, G. M., \& Bouzas, A. (1980). Context dependent changes in the reinforcing strength of schedule-induced drinking. Journal of the Experimental Analysis of Behavior, 33, 327-335.

Innis, N. K., Simmelhag-Grant, V. L., \& Staddon, J. E. R. (1983). Behavior induced by periodic food delivery: The effects of interfood interval. Journal of the Experimental Analysis of Behavior, 39, 309-322.

LeVITSKY, D. \& COLLIER, G. (1968). Schedule-induced wheel running. Physiology \& Behavior, 3, 571-573. 
Penney, J., \& Schull, J. (1977). Functional differentiation of adjunctive drinking and wheel running in rats. Animal Learning \& Behavior, 5, 272-280.

Riley, A. L., Peele, D. B., Richard, K., \& Kulkosky, P. J. (1981). The interaction of conditioned taste aversions and schedule-induced polydipsia: Availability of alternative behaviors. Animal Learning \& Behavior, 9, 488-492.

ROPER, T. J. (1978). Diversity and substitutability of adjunctive activities under fixed-interval schedules of reinforcement. Journal of the Experimental Analysis of Behavior, 30, 83-96.

ROPER, T. J. (1980). Changes in rate of schedule-induced behaviour in rats as a function of fixed-interval schedule. Quarterly Journal of Experimental Psychology, 32, 159-170.

ROPER, T. J. (1981). What is meant by the term "schedule-induced," and how general is schedule induction? Animal Learning \& Behavior, 9, 433-440.

Rosellini, R. A., \& Burdette, D. R. (1980). Meal size and intermeal interval both regulate schedule-induced water intake in rats. Animal Learning \& Behavior, 8, 647-652.

SEGAL, E. F. (1969). The interaction of psychogenic polydipsia with wheel running in rats. Psychonomic Science, 14, 141-144.

Shurtleff, D., Delamater, A. R., \& Riley, A. L. (1983). A teevaluation of the CS - hypothesis for schedule-induced polydipsia under intermittent schedules of pellet delivery. Animal Learning \& Behavior, 11, 247-254.

STADDON, J. E. R. (1977). Schedule-induced behavior. In W. K. Honig \& J. E. R. Staddon (Eds.). Handbook of operant behavior (Vol. 2). New York: Prentice-Hall.

Staddon, J. E. R., \& Ayres, S. L. (1975). Sequential and temporal properties of behavior induced by a schedule of periodic food delivery. Behaviour, 54, 26-49.

Wetherington, C. L. (1979). Schedule-induced drinking: Rate of food delivery and Herrnstein's equation. Journal of the Experimental Analysis of Behavior, 32, 323-333.

WETHERINGTON, C. L. (1982). Is adjunctive behavior a third class of behavior? Neuroscience \& Biobehavioral Reviews, 6, 329-350.

Wetherington, C. L., \& Brownstein, A. J. (1982). Comments on Roper's discussion of the language and generality of schedule-induced behavior. Animal Learning \& Behavior, 10, 537-539.

Wetherington, C. L., Brownstein, A. J., \& ShUll, R. L. (1977). Schedule-induced running and chamber size. Psychological Record, 27, 703-713.

(Manuscript received August 24, 1984; revision accepted for publication February $21,1985$. 